\title{
APPLICATION OF THE RANS FLOW MODEL FOR THE ANALYSIS OF THE MOTION OF FLOATING OBJECTS IN WAVES
}

\author{
Marek Kraskowski \\ Ship Design and Research Centre S.A., Ship Hydromechanics Division \\ Szczecińska Street 65, 80-392 Gdansk, Poland \\ tel.: +48585116348 , fax: +48585531643 \\ e-mail:marek.kraskowski@cto.gda.pl
}

\begin{abstract}
The present paper describes a proposal of the method for simulating the motion of freely floating and moored objects in waves, based on the RANS (Reynolds Averaged Navier-Stokes Equations) flow model. Attempts on using this model for simulating the floating body dynamic started quite recently due to its large requirements for the computer power. Only in the past few years such analyses became more common, however, no unique approach can be observed due to large variety of existing possibilities, numerical methods etc. The paper includes the description of the proposed method, which consists in mesh generation technique, efficient wave generation method, and the mooring system module, as well as two samples of marine applications: analyses of the motion of fishing vessel in head waves and the motion of moored semi-submersible drilling platform. The results were verified basing on the towing tank experiments, conducted by Centrum Techniki Okrętowej (Ship Design and Research Centre) S.A.

Generating the waves by prescribed wave motion on both ends of the domain, generating the waves by prescribed wave motion and suppressing them in the damping zone, sliding mesh example, mooring system scheme, forcedisplacement characteristics of the mooring chain, fishing vessel in head waves, heave motion history, outline of the semi-submersible are presented.
\end{abstract}

Keywords: computational fluid dynamics, ship, drilling platform, mooring, model tests

\section{Introduction}

The problem of a ship hull motion in waves has been successfully analyzed for decades with the use of simplified mathematical models, especially the so-called strip theory. Although the simplified models are capable of providing with relatively accurate results at very low computational effort, their range of application is considerably limited due to the assumed simplifications. The problem concerns especially the analysis of the objects of quite different shape than typical ship hulls, as well as direct simulation of the phenomena occurring during violent motion, e.g. green water on deck or slamming. The RANS flow model, based directly on mass and momentum conservation equations, is by definition free of the mentioned limitations; however, its computational cost is incomparably higher. Nevertheless, increasing computational power of CPU's as well as the need for universal computational models result in extending applicability of RANS-based models in the analysis of floating objects.

The work presented here is an attempt at practical application of the RANS-based method for the analysis of freely floating and moored objects' response to sea waves. A technique for efficient meshing has been proposed, as well as a method for accurate regular wave generation. Additionally the influence of the mooring system has been included in the computational model.

The STAR CCM+ software was used for all computations, and the free surface problem was solved using the volume of fluid (VOF) model; the computational results were verified basing on the results of standard model tests, conducted in the towing tank. In order to enable direct comparison of computational and experimental results, the computations were carried out at the model scale. 


\section{Simulating the wave motion in RANS computations}

The most common procedure of analysing the floating object's response to waves is based on the measurements of its motion in irregular wave of given spectrum. The measured trajectory undergoes a spectral analysis, resulting in statistic parameters of the object's seakeeping in particular operating region (significant amplitudes of roll, pitch, heave etc.)., It was decided not to reproduce exactly this well-established experimental approach in the proposed method due to the following reasons:

- obtaining reliable results requires relatively long time of exposing the analyzed object to irregular wave, which means extremely long computational time for one case;

- controlling the correctness of generating the irregular wave is much more complex than for regular wave;

- both the advantages of the RANS model (no crude simplifications) and its disadvantages (low time efficiency) predetermine it rather for simulating some selected interesting situations (e.g. extreme sea states) than for complete, standard seakeeping analysis.

The simulations made so far are then based on regular waves only; however simulating the regular waves in RANS flow model is not a trivial task also. The following requirements should be fulfilled in order to assure the accuracy of the computational model:

- the generated waves should maintain their parameters (height and frequency) throughout the simulation;

- the waves introduced to the computational domain should leave it without generating any nonphysical reflections that may disturb the determined wave motion.

Generating the waves is in itself relatively simple and is usually realized by setting up proper boundary condition: an unsteady velocity profile and water elevation is prescribed at one of the domain boundaries ("inlet"). Both the velocity profile and wave elevation can be based on the linear wave theory, although it is valid only for the waves of very low height/length ratio; for steeper waves, the resulting wave will turn into a realistic wave anyway, if only a periodic disturbance is introduced to the flow. It is more important and difficult to assure that no wave reflections will occur on the opposite side of the computational domain - "outlet" (this is also a problem to be solved in the experiment in a towing tank). In the RANS computations, this can be realized in a few ways:

- at the outlet boundary, the wave elevation and velocity profile can be also prescribed, taking into account the phase shift, allowing the wave to leave the domain (Fig. 1); such an approach is the simplest one and allows using compact computational domain, however, it is not completely free from the mentioned above problems: the wave reaching the outlet boundary (elevation and velocity profile) is always somewhat different from the theoretical wave, especially in case of using the linear theory, moreover, it is disturbed by the floating object. In such case, certain "conflict" occurs at the outlet boundary due to difference between the actual and theoretical parameters of the wave leaving the domain, resulting in disturbance of the required flow. It can be accepted in particular cases, especially if the simulation time is not longer than only few periods of the wave,

- a damping zone can be introduced downstream from the analyzed object, in some distance upstream of the outlet boundary, in which the mesh density is being gradually reduced, making it insufficient for simulating the wave motion of water; as a result, the flow reaching the outlet boundary becomes uniform and can easily leave the domain without producing nonphysical reflections (Fig. 2). Prescribed hydrostatic pressure is a sufficient boundary condition for the outlet. Major disadvantage of this approach is that the domain length (and also the number of mesh cells) must be substantially extensive to assure proper damping of the wave motion,

- in alternative approach, also taking the advantage of wave damping zone, the artificial damping of the vertical motion is accomplished by introducing additional term to the momentum 
equation instead of reducing the mesh density. In this approach, the damping zone size can be considerably reduced, which allows for a reasonable compromise between stability of the computations and the computational time. This method has been used in the present work.

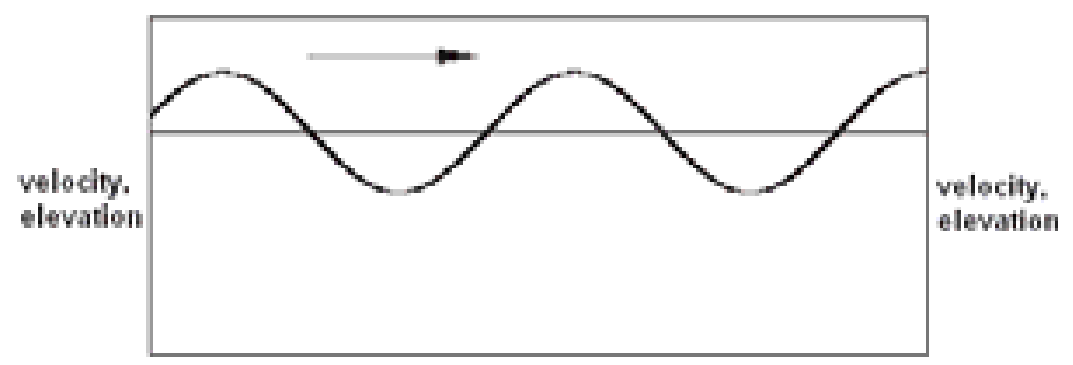

Fig. 1. Generating the waves by prescribed wave motion on both ends of the domain

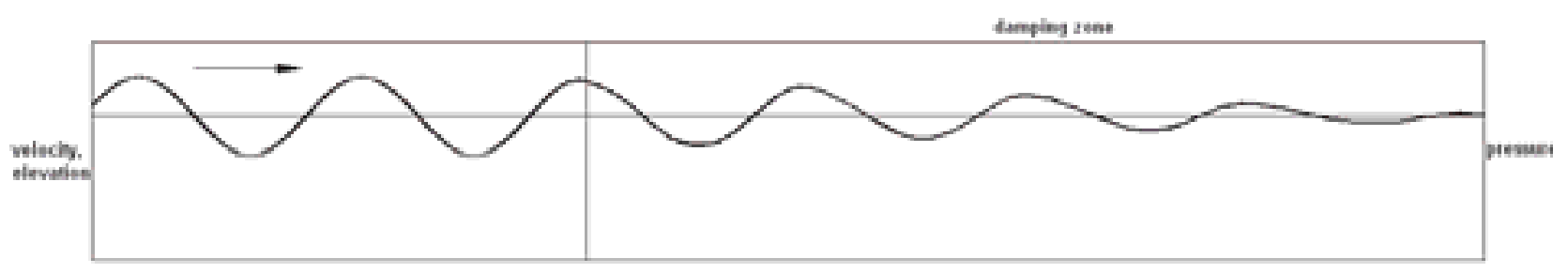

Fig. 2. Generating the waves by prescribed wave motion and suppressing them in the damping zone

\section{Meshing technique for the floating body analysis}

The computational mesh, in which the flow equations are solved (equation of state, mass and momentum conservation equations) must move together with the moving object what stands for an important problem in RANS simulations of their movement. Different approaches are possible in this case also. The choice of moving mesh type for particular type of simulation including fluidstructure interaction depends on pros and cons of these types; the most popular approaches are (see Fig. 3):

- rigid mesh moving together with moving object (no relative motion between mesh nodes) simplest possible approach, characterized by lowest computational cost and fairly appropriate in cases like estimation of dynamic trim and sinkage of the vessel in calm water. However, the accuracy of this approach rapidly decreases for high motion amplitudes, especially large angles, due to violent motions of the domain boundaries,

- deforming mesh [1] - characterized by high accuracy, but appropriate only for cases where motion amplitudes are relatively low. Too large displacement of the object from its initial position causes unacceptable deformation of the mesh cells,

- sliding mesh [2] - the flow domain is divided in two subdomains; the inner domain, surrounding the analyzed object, is moving together with it, and the outer domain undergoes only linear motions. This approach is characterized by much higher tolerance for large amplitudes of angular motions than the rigid mesh, but also considerably higher computational cost,

- overlapping meshes - robust and quite accurate, but not available in current version of the software used for the analysis.

The goal of the reported research was to elaborate a robust computational method capable of handling large angular motion amplitudes, so the choice of sliding meshes for the computations of floating objects in waves was considered an optimum solution among the options available in STAR CCM+ solver. An example of real working sliding mesh, used in reported work, is presented in Fig. 4. 


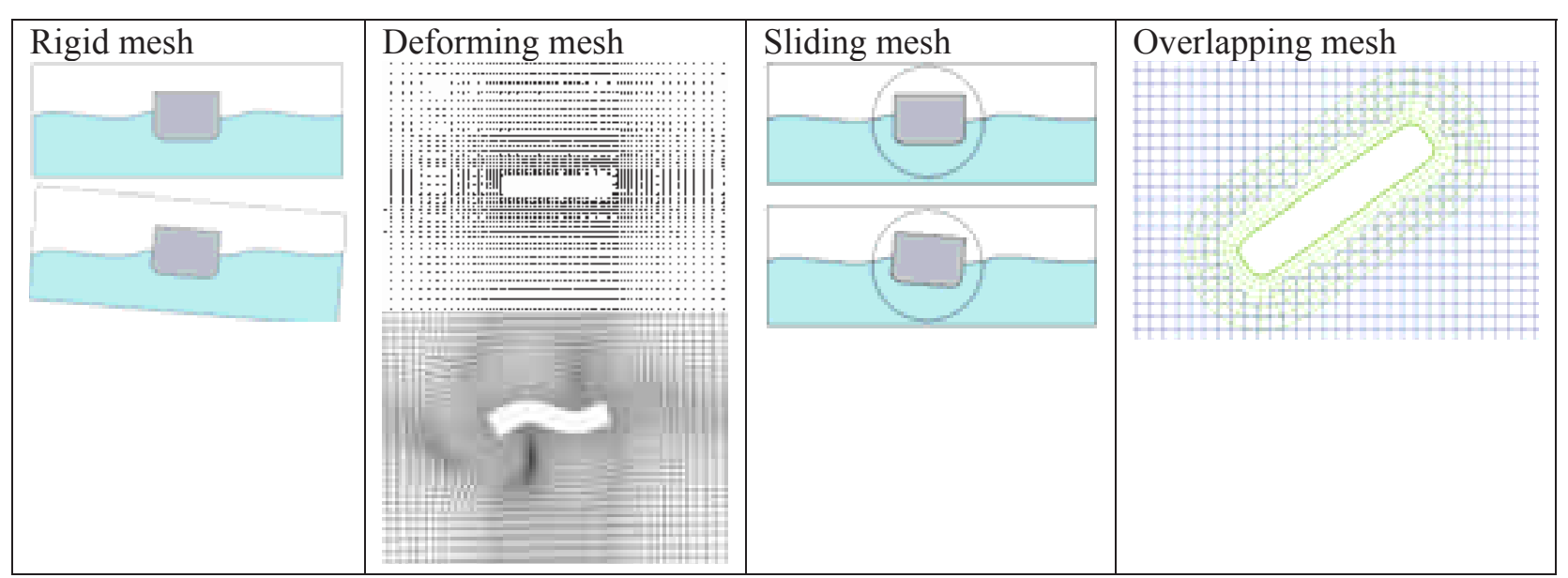

Fig. 3. Types of mesh

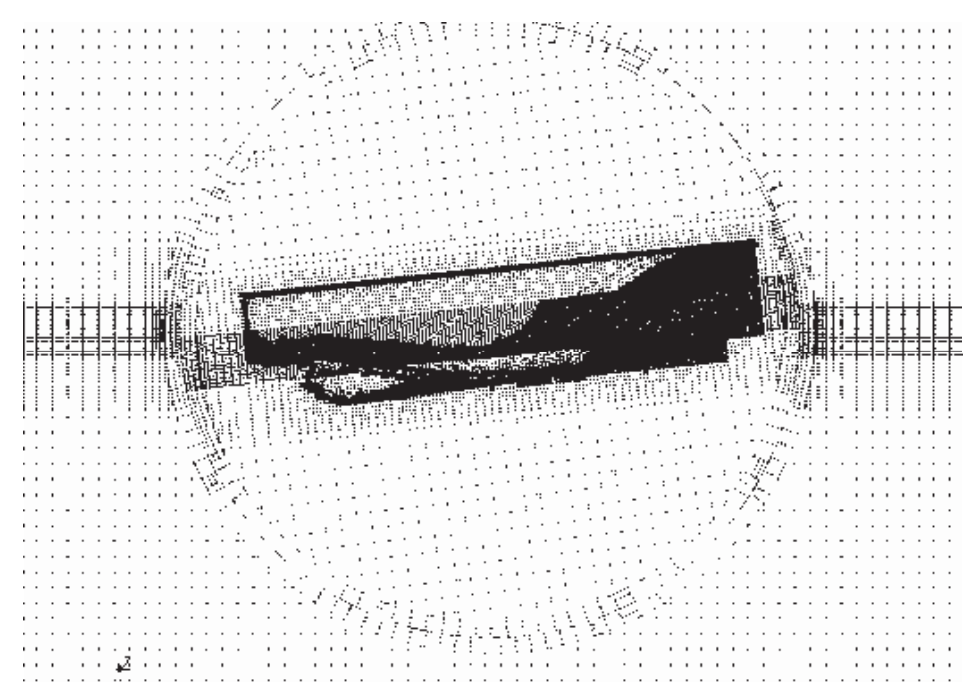

Fig. 4. Sliding mesh example

\section{Method of simulating the mooring system}

Theoretically, it is possible to carry out a coupled simulation including both the floating object and a finite element representation of the mooring chains (or lines), in which two-way interaction between the mooring and the object is considered. However, whenever possible, it is much more economical to model the mooring system by replacing the mooring chains with equivalent forces, dependent on the object motion parameters: displacement, velocity and acceleration. Proper identification of the mooring chain characteristics is then necessary, using the additional software. This approach was used in the presented work.

A simplifying assumption has been made at this stage, that from the point of view of the floating object motion characteristics, it is sufficient to include only the static characteristics of the mooring system (i.e. that the forces exerted on the object depend only on the object displacement). This means that the mooring system characteristics determine the mean drift of the moored object, but that they do not influence its wave frequency motion. Validity of this assumption (made on the basis of preliminary literature studies) will undergo further analysis. It is obvious that the forces in the mooring chain increase with growing frequency of the object motion, and it is necessary to take this effect into account in the analysis of the chain strength, but this is not the point of interest in the present work.

A program allowing investigating of the mooring system characteristics was developed basing on the lumped mass method, proposed by Nakajima, Motora and Fujino [3]. The algorithm of the method is described in detail in the cited paper, so just a brief outline is given here: the chain is approximated 
by a set of point masses connected with linear springs, the end point undergoes a prescribed motion and the tension distribution along the chain is iterated in each time step so as to fulfil the equilibrium conditions. Added mass and resistance of each element of the chain are taken into account.

The typical mooring system of a semi- platform usually involves the chain/wire elements; however, complex systems involving buoys, weights and rigid elements are also in use. The characteristics of such systems can be identified only computationally. For the purpose of test computations of semi-submersible platform, a simple mooring system was assumed, consisting of eight uniform chains (Fig. 5). Static characteristics of the mooring chain forming a catenary can be calculated analytically [4], what makes simple validation of the numerical method possible. The force-displacement characteristics, calculated both numerically and analytically, are presented in Fig. 6; satisfactory agreement was achieved.

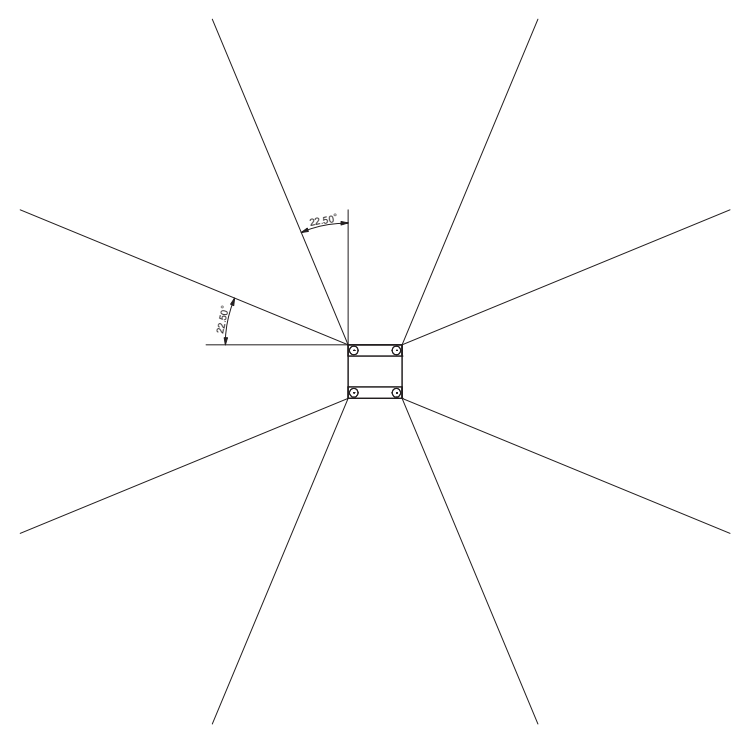

Fig. 5. Mooring system scheme

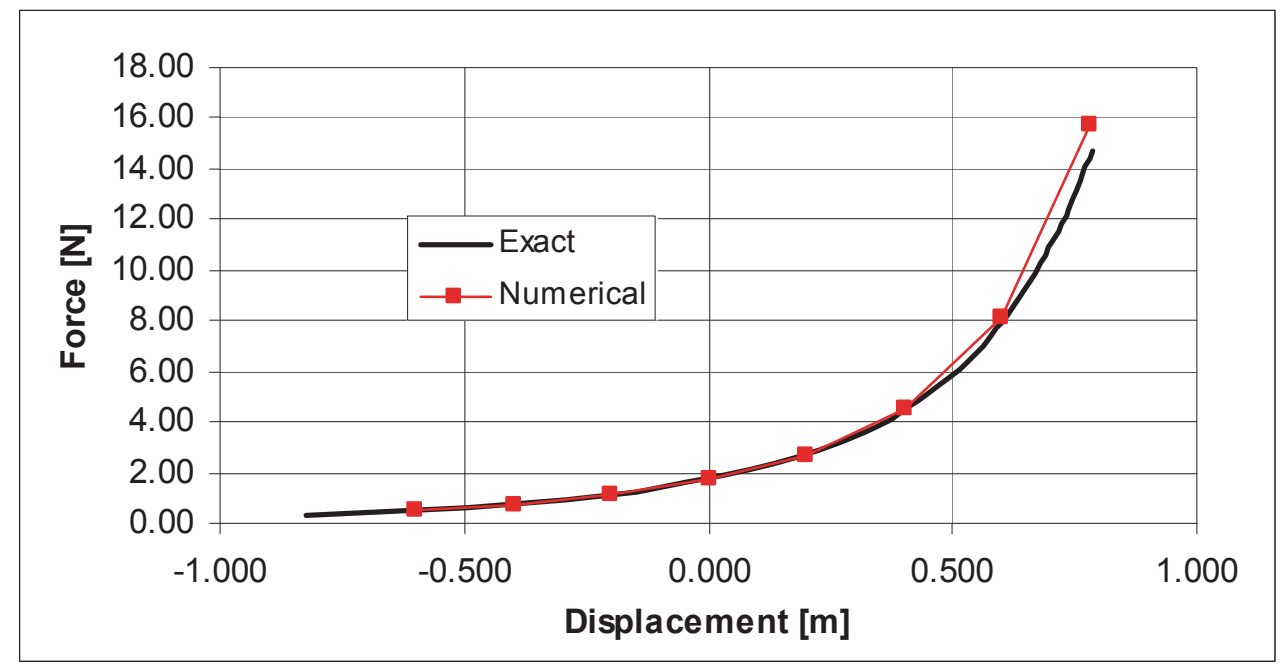

Fig. 6. Force-displacement characteristics of the mooring chain

\section{Results of the test computations and validation}

The proposed method for simulating the floating body motion in waves was initially verified basing on two test cases: a fishing vessel sailing at constant speed in head waves, and a moored 
semi-submersible platform in quartering waves. The results of computations were compared with the results of towing tank tests.

The fishing vessel case includes the computations for three regular waves; their $\lambda$ lengths correspond to $0.98,1.31$ and 1.57 of the vessel's length $L$. Fig. 7 presents the vessel in medium length wave. The method of generating the waves involves prescribed wave motion on both ends of the computational domain. The domain length was $5 L$, with the vessel located approximately in the middle; the number of mesh cells was 830000 .

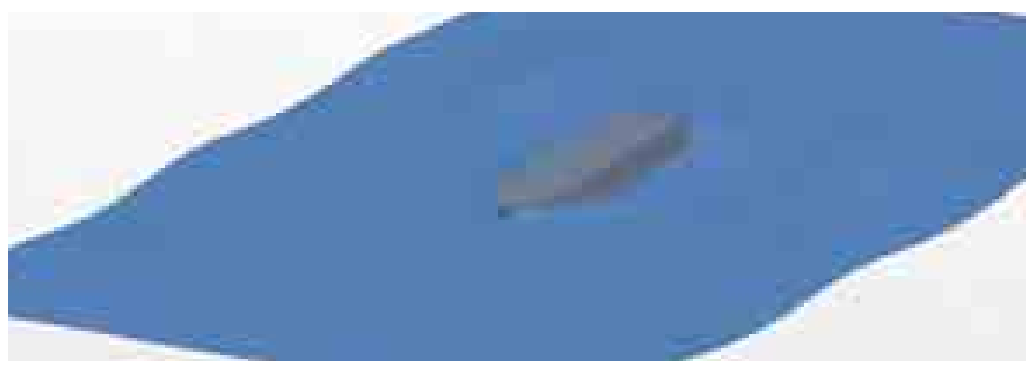

Fig. 7. Fishing vessel in head waves

Sample results are presented below (Fig. 8-10): the time history of the heave motion (tRANSlational motion of the vessel's centre of gravity along the vertical axis); the results of computations (black line) are compared with the experimental results (red line).

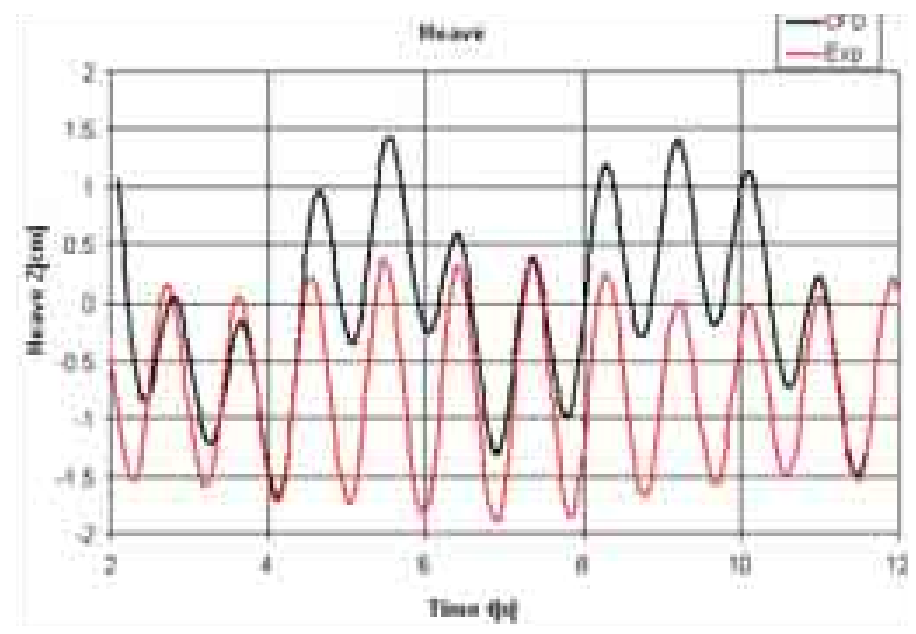

Fig. 8. Heave motion history - the shortest wave, $\lambda / L=0.98$

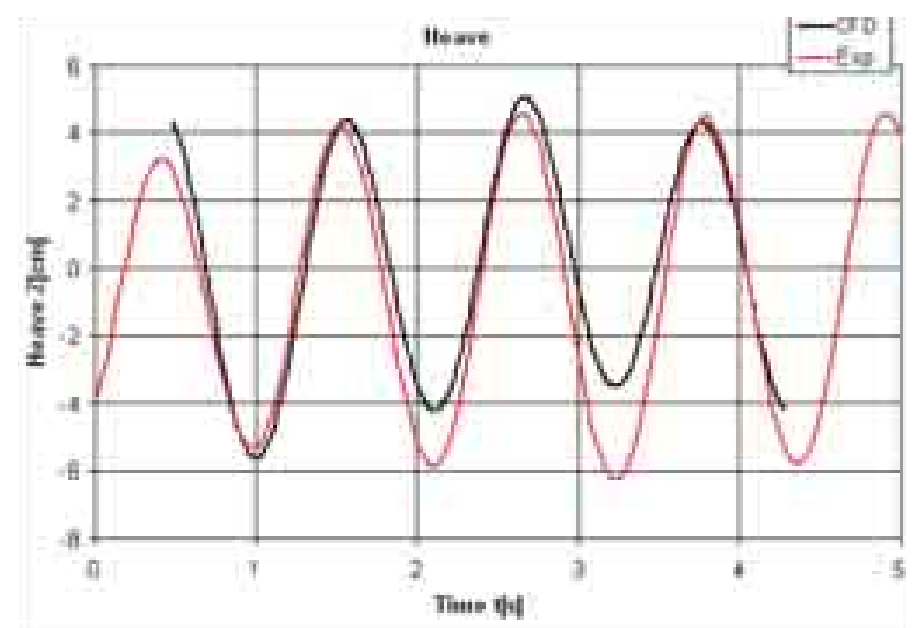

Fig. 9. Heave motion history-medium wave, $\lambda / L=1.31$ 


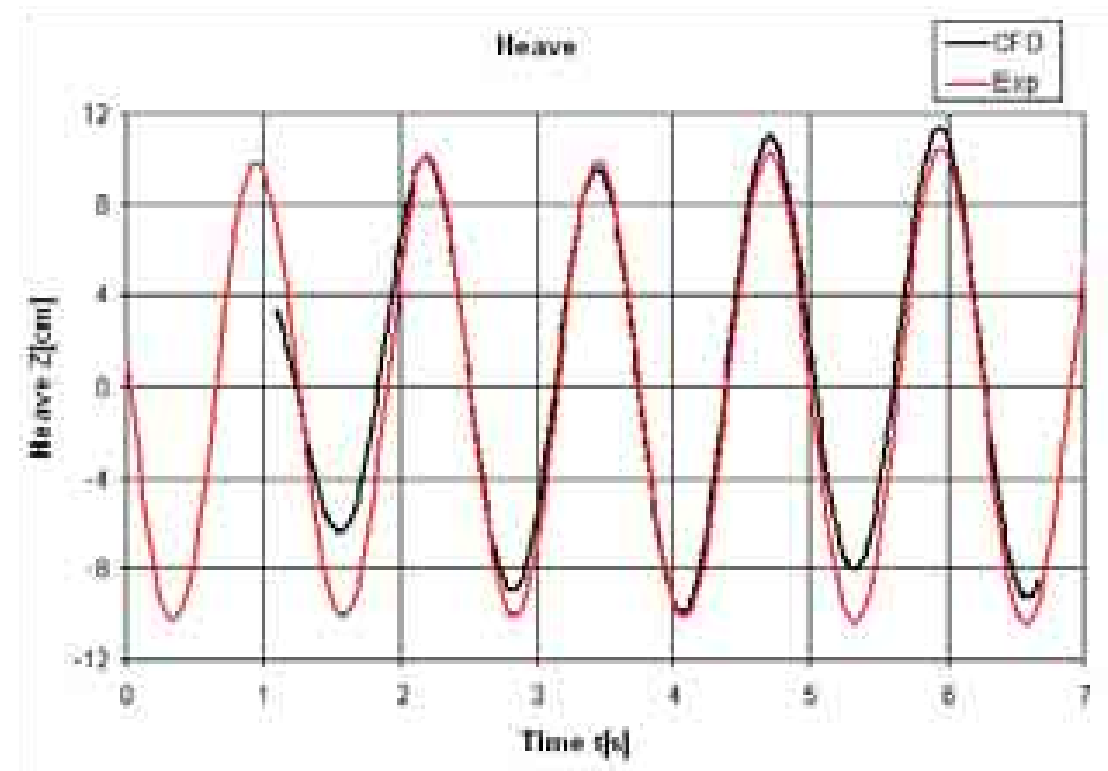

Fig. 10. Heave motion history - the longest wave, $\lambda / L=1.57$

Clearly, the most stable and accurate results were achieved for the longest wave (Fig. 9). For the shortest wave, strong oscillation of the solution can be observed, which is most probably due to nonphysical oscillation of the generated wave due to wave reflection at the domain outlet, described in section 2. The approach to wave generation involving prescribed wave motion at both ends of the domain was then abandoned, and artificial wave damping was used instead in further tests with semi-submersible platform.

The simulation of motion of the semi-submersible platform was realized for only one case: regular wave of relative length $\lambda / L=1.85$ (the characteristic length $L$ of the platform was marked in Fig. 11). The domain length was $7 L$, while the length of the damping zone was $3.2 L$. The number of mesh cells was app. 2000000 . The measured time histories of motion were unavailable for this case, therefore only the so-called response amplitude operators (RAOs) will be used for comparison with computations. Response amplitude operator RAO, characterizing the object's response to wave of given frequency, is defined as the amplitude of periodic motion in selected degree of freedom, normalized by wave amplitude (tRANSlational motion) or wave slope (angular motion). Comparison of computed and measured RAOs is presented in Tab. 1.

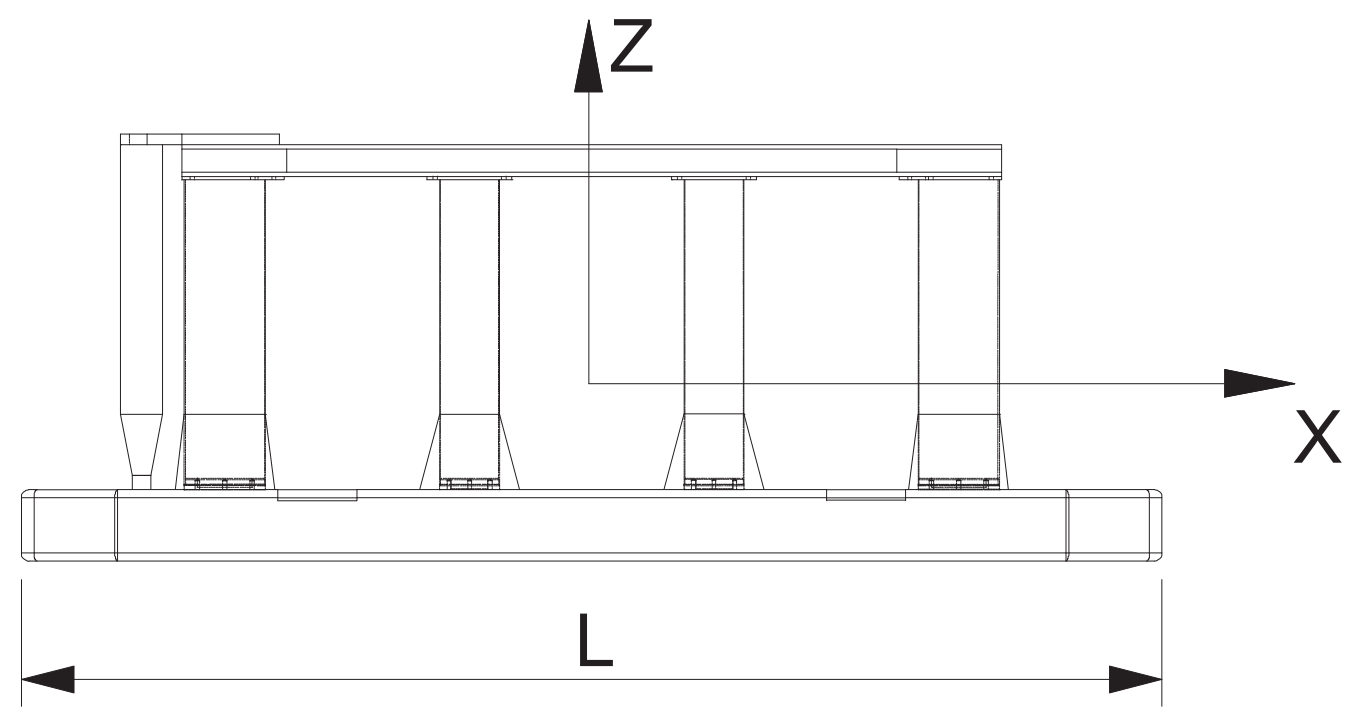

Fig. 11. Outline of the semi-submersible platform, with marked coordinate system originated at centre of gravity 
Tab. 1. Comparison of computed and measured RAOs for semi-submersible platform

\begin{tabular}{|l|c|c|c|}
\hline & Surge RAO [ - ] & Heave RAO [ - ] & Pitch RAO [ - ] \\
\hline Experiment & 0.239 & 0.387 & 0.251 \\
\hline Computations & 0.284 & 0.340 & 0.304 \\
\hline Error [\%] & 18.9 & -12.2 & 21.0 \\
\hline
\end{tabular}

The discrepancies between the computations and the experiment for this first analyzed case are quite large and difficult to explain at this stage; they can be caused either by inaccurate flow modelling, or by simplifications in modelling the mooring system. For that reason, more computational cases will be analyzed and the results will be compared with the results of the experiment dedicated especially for validation purposes. However, the computational results can be considered realistic anyway, and characterized by correct tendency (the highest RAO for the heave motion, the lowest - for the surge motion).

\section{Conclusions and further work indications}

Basing on the results obtained and observations of convergence of the computations, the following conclusions can be done:

- the proposed method of analysing the floating body motion, based on RANS flow model and sliding mesh technique, is characterized by sufficient robustness and is capable of producing relatively accurate results for some of analyzed cases,

- problems with stability and accuracy, observed in the analysis of fishing vessel in shortest wave, can be explained by nonphysical wave reflection within the domain; the modified approach to wave generation, involving the damping zone, will probably allow to overcome these problems,

- analysis of the semi-submersible platform revealed very long convergence time (time to reach periodic oscillations) due to high disturbance at the beginning of the simulation, before the mooring system reached its equilibrium condition. An artificial damping will be then used at the beginning of the simulation in further tests, in order to speed up the convergence,

- the results of the simulation for semi-submersible platform, involving the mooring system, are realistic, but not accurate enough. Further model tests, designed especially for validation purposes, will be carried out, followed by equivalent numerical simulations, in order to identify the problems and, hopefully, improve the accuracy.

\section{References}

[1] Oberhagemann, J., el Moctar, O., A Simplified Approach to Investigate Fluid-Structure Coupling Effects on Slamming Loads of Ships, Numerical Towing Tank Symposium, Hamburg 2007.

[2] Kraskowski, M., Analysis of the floating structures using sliding meshes, Numerical Towing Tank Symposium, Poole 2011.

[3] Nakajima, T., Motora, S., Fujino, M., On the Dynamic Analysis of Multi-Component Mooring Lines, $14^{\text {th }}$ Offshore Technology Conference, 1982.

[4] Baltrop, N. D. P., et al., Floating structures: a guide for design and analysis, The Centre for Marine and Petroleum Technology, 1998 